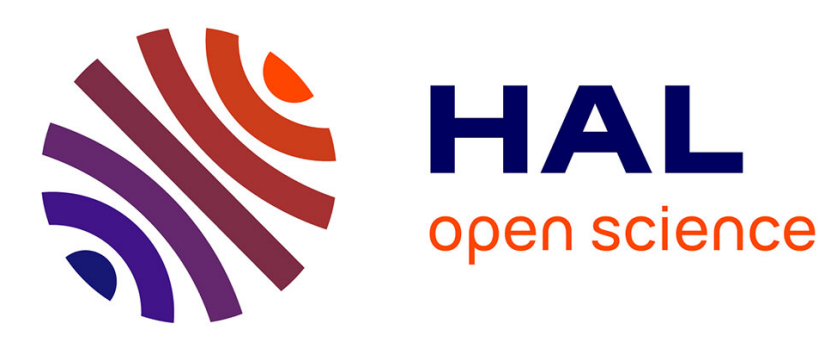

\title{
La ligne à grande vitesse californienne : entre mythes et réalités
}

\author{
Charlotte Ruggeri
}

\section{To cite this version:}

Charlotte Ruggeri. La ligne à grande vitesse californienne : entre mythes et réalités. RTS Recherche Transports Sécurité, 2014, Grande vitesse ferroviaire et territoires, 2013 (03), pp.229-244. 10.4074/S0761898013003063 . hal-01670632

\section{HAL Id: hal-01670632 \\ https://hal.science/hal-01670632}

Submitted on 21 Dec 2017

HAL is a multi-disciplinary open access archive for the deposit and dissemination of scientific research documents, whether they are published or not. The documents may come from teaching and research institutions in France or abroad, or from public or private research centers.
L'archive ouverte pluridisciplinaire HAL, est destinée au dépôt et à la diffusion de documents scientifiques de niveau recherche, publiés ou non, émanant des établissements d'enseignement et de recherche français ou étrangers, des laboratoires publics ou privés. 


\title{
La ligne à grande vitesse californienne : entre mythes et réalités
}

\author{
The High-Speed Rail in California: between myths and realities
}

\author{
Charlotte Ruggeri
}

Reçu le 29 septembre 2012 ; accepté le 3 octobre 2013

(C) IFSTTAR et Éditions NecPlus 2013

\begin{abstract}
Résumé Depuis 1993, l'État californien développe un discours et un projet ambitieux de ligne à grande vitesse entre Sacramento et San Diego, en passant par les deux principales métropoles de l'État que sont San Francisco et Los Angeles. Ce projet de grande vitesse californienne, qui doit desservir 24 villes et dont les premiers tronçons seront inaugurés en 2022, nous interroge sur les raisons et les conditions d'élaboration d'une telle infrastructure dans un pays où la grande vitesse ferroviaire est presque absente. Il nous invite à analyser les potentiels impacts spatiaux de cette infrastructure sur les territoires desservis, en termes de hiérarchie urbaine, de rapports entre les acteurs territoriaux (État californien, comtés, villes) et enfin d'appropriation et d'ancrage de la nouvelle infrastructure dans les villes. Quelles mutations - tant au niveau des pratiques que des territoires - la ligne à grande vitesse peut-elle engendrer sur les centres urbains californiens ? Les premières recherches sur le terrain montrent des profils variés d'acteurs locaux : soit engagés dans des politiques ambitieuses de restructuration urbaine, soit relativement indifférents au projet. Le discours de l'acteur principal (la California High-Speed Rail Authority) illustre l'ambition de dessiner une Californie de prestige et d'image qui contraste avec la réalité géographique et politique.
\end{abstract}

Mots clés train à grande vitesse $\cdot$ territoire $\cdot$ restructuration $\cdot$ réseau urbain $\cdot$ mobilité

\begin{abstract}
Since 1993, the State of California has communicated on the ambitious project to create a high-speed rail network between Sacramento and San Diego, through San Francisco and Los Angeles. The high-speed rail in California should connect 24 cities and the first tracks will open in 2022. Why and how this infrastructure is to be
\end{abstract}

Charlotte Ruggeri $(\bowtie)$

Université de Cergy Pontoise

EA 4113 - Laboratoire MRTE

33 boulevard du Port, 95011 Cergy-Pontoise cedex

charlotte.ruggeri@gmail.com developed in a country where high-speed rail is almost non-existent? This research is a survey of the spatial impacts of the high speed rail project on cities, urban centers, urban network, urban mobility practices, institutional structures and actors (cities, counties and state). What changes could high-speed rail induce onto practices and territories, especially in Californian downtonws. At this early stage of field research the main players' attitude vary from active support for ambitious urban redevelopment actions to indifference. For instance the California High-Speed Rail Authority has communicated on its vision of prestige for California which contrast with the actual geographical and political reality.

Keywords High-speed rail · Territory · Redevelopment . Urban network · Mobility

En 2010, Barack Obama annonce la construction de 13 corridors à grande vitesse aux États-Unis. Depuis ce discours, peu de projets ont vu le jour et certains projets, notamment en Floride, ont été abandonnés. La Californie résiste et a probablement le projet le plus avancé de ligne à grande vitesse ferroviaire. Sur 1200 kilomètres, l'État californien entend desservir ses principales métropoles (Sacramento, San Francisco, Los Angeles et San Diego), ainsi que 18 villes secondaires du réseau urbain. Les travaux devraient commencer en 2013 au nord de l'État. Si le projet californien se maintient et a des chances de voir le jour dans un pays peu prompt à la construction de grandes infrastructures publiques de transport, cela est également dû à une volonté politique forte de la part du gouverneur Jerry Brown qui veut faire du train à grande vitesse un « héritage » pour les générations futures. Ce projet est piloté par une agence régionale publique, la California High-Speed Rail Authority (CHSRA).

Cette volonté et ce projet (qui devrait être mis en service entre 2022 et 2035) interrogent les raisons et les conditions d'élaboration d'une telle infrastructure publique dans un pays où la grande vitesse ferroviaire est très peu développée. Ce projet n'a pas d'équivalent aux États-Unis et les acteurs locaux doivent penser et préparer l'arrivée de la 
grande vitesse ferroviaire sans références nord américaines. Comment ces territoires accueillent-ils ce projet, que ce soit d'un point de vue politique ou spatial ? Dans un territoire vierge de la grande vitesse, les acteurs territoriaux sont face à une aubaine qui peut se décliner en une multitude de stratégies locales. La grande vitesse est-elle un prétexte pour restructurer les territoires locaux californiens et un catalyseur de projets d'aménagement du territoire à l'échelle urbaine?

La conception du réseau du futur train à grande vitesse californien est celle d'un réseau multiscalaire qui connecte et articule plusieurs échelles et différents réseaux (que ce soient des réseaux urbains ou de transports). Au sommet, l'échelle régionale construit et défend le discours rhétorique du projet de ligne à grande vitesse, en creux duquel on observe la conception d'une certaine représentation du territoire californien, tournée vers l'innovation territoriale. À l'échelle infrarégionale et urbaine, cette nouvelle desserte ferroviaire pourrait engendrer une concurrence entre les acteurs territoriaux (État californien, comtés, villes) en termes d'appropriation de l'infrastructure. Enfin, à l'échelle infra-urbaine, la ligne à grande vitesse interroge le modèle de la ville américaine puisqu'elle pourrait engendrer une restructuration des centres urbains dans un territoire avant tout périurbain. Le projet de ligne à grande vitesse californienne pose donc la question de l'ancrage territorial d'une nouvelle desserte ferroviaire sur le territoire nord-américain, très peu habitué à ce type d'infrastructure.

Les réalités géographique, sociale et politique (observée lors de deux périodes de travail de terrain en Californie ${ }^{1}$ ) ne semblent pas favorables à cette nouvelle infrastructure ferroviaire. Nos enquêtes individuelles et collectives mettent en évidence des acteurs impliqués, mais aussi une population californienne qui exprime une relative indifférence vis-à-vis de ce projet très peu connu du public (au vu des réactions négatives constatées dans des réunions publiques ${ }^{2}$ ). Étant donné la difficulté de cerner l'opinion publique sur ce projet, cet article se propose d'analyser son acceptation et son appropriation par les acteurs publics, notamment les municipalités, les comtés et les Metropolitan Planning Organizations $\left(\mathrm{MPO}^{3}\right)$. Une nouvelle desserte ferroviaire représente des potentialités pour les territoires locaux dont la concrétisation dépend de la volonté et de l'action des acteurs. L'appropriation relève alors de la capacité de ceux-ci à se saisir de ces potentialités [1]. Une nouvelle desserte ferroviaire permet

\footnotetext{
${ }^{1}$ Séjours réalisés en 2010-2011 et en 2012.

${ }^{2}$ Ces réunions sont organisées avant tout par la CHSRA.

${ }^{3}$ Les MPO sont des agences publiques métropolitaines chargées de planifier, financer et aménager les politiques publiques de transports ainsi que de coordonner les opérateurs de transport sur un territoire urbain donné.
}

une amélioration de l'accessibilité des territoires desservis et suscite aussi des attentes économiques. Toutefois, leur concrétisation dépend en grande partie de la mise en œuvre de stratégies locales [2]. Cette appréhension politique de la ligne à grande vitesse ferroviaire permet de construire une réflexion sur l'ancrage territorial et géographique que cette future infrastructure pourrait avoir dans les métropoles californiennes, notamment en termes de restructuration urbaine.

L'étude du discours politique sur la ligne à grande vitesse californienne s'incrit dans un contexte nord-américain en apparence peu favorable à la grande vitesse ferroviaire, mais laisse entrevoir une certaine conception du territoire californien construite par la CHSRA et l'État californien. Au-delà de ce discours, cet article tente de montrer la réalité des mutations et des évolutions de cette nouvelle desserte ferroviaire sur le territoire. Mutations inédites pour les acteurs territoriaux et pour les habitants en termes d'échelles, d'acteurs, de réseaux et de hiérarchie urbaine. En effet, l'ambition du futur réseau à grande vitesse est de s'affirmer comme une strate supérieure du territoire californien qui restructure et articule les réseaux urbains et de transports existants. Enfin, l'étude précise des réhabilitations urbaines en cours permet de mettre en avant l'idée d'acteurs territoriaux qui profitent du projet de la grande vitesse pour penser une restructuration urbaine des quartiers des futures gares.

\section{La construction d'un discours : le projet de ligne à grande vitesse en Californie}

Le projet actuel de grande vitesse californienne naît en 1993 avec la création par l'État californien de l'Intercity HighSpeed Rail Commission [3]. Pour comprendre les raisons d'un tel retard dans la grande vitesse depuis la génèse de cet ambitieux projet, les contextes nord-américain et californien doivent être pris en compte ainsi que leurs évolutions actuelles qui semblent redevenir favorables à la grande vitesse comme l'illustre la multiplication récente de projets.

\section{Le contexte : la grande vitesse aux États-Unis}

Avant d'étudier la grande vitesse californienne, il convient de s'interroger sur la grande vitesse américaine. En France, la grande vitesse, malgré ses difficultés initiales, est aujourd'hui un acquis et un fait banal, mais peu d'autres pays affichent un soutien et une foi aussi forte envers cette infrastructure [4]. Le chemin de fer a été développé aussi précocement aux États-Unis qu'en Europe, puisque les premières voies de chemin de fer apparaissent dans les années 1830. Le mythe du grand Ouest américain, du Far West, et donc entre autres de la Californie, se construit 
autour de cette symbolique du chemin de fer qui achemine des hommes prêts à faire fortune de l'Est des États-Unis. Dès 1862 , l'État récemment intégré à l'Union américaine est relié par une voie de chemin de fer transcontinentale de 3200 kilomètres qui part de Sacramento vers l'Est [5]. Avec le chemin de fer, le tourisme se développe rapidement dans cet État ensoleillé plus de 200 jours par an. Les compagnies ferroviaires du sud de la Californie (la Southern Pacific et la Santa Fe Railroad) développent alors un argumentaire publicitaire vantant les mérites du climat californien, des vacances dans le sud de la Californie, mais aussi des paysages grandioses des parcs nationaux [6].

Toutefois, malgré cette railwaymania précoce, les ÉtatsUnis, comme les autres pays développés, n'engagent plus de développement ambitieux de leur réseau ferré après la Seconde Guerre mondiale [4]. Les investissements fédéraux sont alors destinés aux infrastructures routières, autoroutières et aériennes. Le rail n'est pas totalement oublié avec la création d'Amtrak (contraction de American Travel on Track) par le Congrès en 1970. Amtrak est une entreprise publique dont le but est de venir en aide aux compagnies ferroviaires dont la fréquentation de passagers déclinait nettement depuis la fin de la Seconde Guerre mondiale. La nouvelle société publique entre en service en 1971 [4].

Parallèlement, le Congrès instaure l'Office of HighSpeed Ground Transportation qui a pour mission d'étudier l'implantation possible d'un corridor ferroviaire à grande vitesse entre Washington DC, New York et Boston [7]. Le Northeast Corridor (NEC) voit le jour en 1988 et devient l'Acela Express en $2000^{4}$ [8]. Dans ce contexte, Amtrak et le département d'État aux Transports étudient d'autres corridors potentiels, notamment en Floride, au Texas et en Californie entre Los Angeles et San Diego. Ces trois projets sont abandonnés à la fin des années 1980 ou au début des années 1990 en raison du refus du gouverment fédéral ou des États de participer aux investissements financiers ou du fait de l'absence d'investisseurs privés [4].

\section{La naissance du projet californien}

Pourtant, quelques années plus tard en 1993, l'État californien fonde une commission (Intercity High-Speed Rail Commission) dont la mission est de créer une agence publique régionale chargée de réfléchir à un projet de ligne à grande vitesse en Californie. La California High-Speed Rail Auhtority (CHSRA) est fondée en 1996 et reçoit le soutien de nombreuses villes, comtés et agences publiques de transport [3]. Les premières études d'impact sont menées dès 2001 et le premier projet est dévoilé en 2004. En 2008,

\footnotetext{
${ }^{4}$ L'UIC (Union internationale des chemins de fer) inclut l'Acela Express dans sa liste des lignes à grande vitesse dans le monde de 2009 [9].
}

les Californiens approuvent le projet lors du vote de la Proposition 1A qui permet à l'État californien de débloquer des fonds ${ }^{5}$. En 2012, le Sénat californien approuve le budget présenté par le gouverneur Jerry Brown ${ }^{6}$.

Le train à grande vitesse californien a initialement été conçu pour relier les deux principales métropoles de l'État-San Francisco au Nord et Los Angeles au Sud — avec un train à 220 miles par heure (soit 350 kilomètres par heure). Ce projet a été revu à la hausse avec une liaison vers le Nord jusqu'à Sacramento (capitale fédérale) et vers le Sud jusqu'à San Diego. Le tracé s'étend sur un peu plus de 1200 kilomètres et se décline en 24 gares. Il dessert 10 gares principales qui sont situées dans les principales agglomérations californiennes. 14 gares intermédiaires (présentées comme des " arrêts » par la CHSRA) sont également envisagées [3]. Le réseau à grande vitesse ne s'étend pas jusqu'au nord de l'État, très peu urbanisé. Cette ligne à grande vitesse s'aligne en grande partie sur les lignes ferroviaires Amtrak préexistantes et prévoit donc un maillage assez serré du sud du territoire californien autour des métropoles de San Francisco, Los Angeles et San Diego.

\section{Un contexte de nouveau favorable à la grande vitesse ?}

La première évolution de la grande vitesse dans les années 2000 aux États-Unis consiste en l'amélioration du NEC qui devient l'Acela Express en 2000. Ce succès aurait ouvert « l'appétit» des Américains pour la grande vitesse [10]. Le projet californien reprend de la vigueur à partir de 2009 à la suite d'un discours de Barack Obama annonçant une subvention fédérale de 8 milliards de dollars pour treize corridors à grande vitesse ${ }^{7}$ [11]. La Californie en est le plus important bénéficiaire, avec des fonds de 2,34 milliards de dollars [12].

La ligne à grande vitesse californienne s'inscrit dans un contexte de renouveau de la grande vitesse aux États-Unis. Le discours de Barack Obama a relancé, voire initié, plusieurs projets [13]. On peut identifier huit projets de grande vitesse aux États-Unis aujourd'hui, dont l'avancement est très contrasté. Certains projets, comme la ligne californienne ou le Master Plan du NEC, sont très avancés et ont une chance de voir le jour [14] [15]. D'autres projets, comme les quatre corridors du Midwest [16] ou l'Xpress West entre Las Vegas et Los Angeles [17],

\footnotetext{
${ }^{5}$ La Proposition 1A, votée le 4 novembre 2008, s'intitule Safe, Reliable High-Speed Passenger Train Bond Act for the $21^{\text {st }}$ Century.

${ }^{6}$ Voir l'article de Judy Lin paru le 6 juillet 2012 dans le Huffington Post-San Francisco, « California High Speed Rail Funding Approved ».

${ }^{7}$ Voir la page du site de la Maison Blanche www.whitehouse.gov/high-speed-rail.
} 


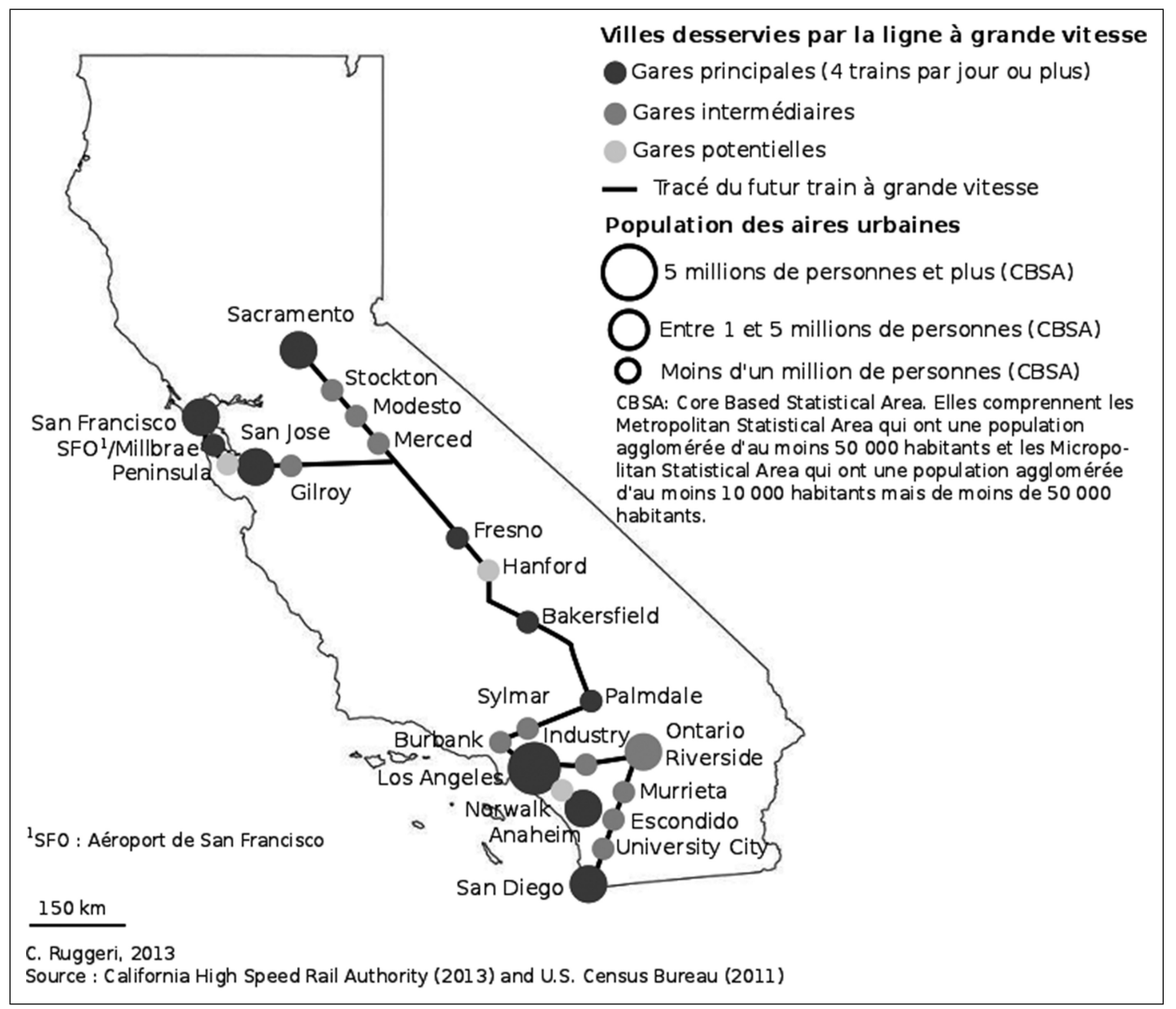

Fig. 1 Le projet de ligne à grande vitesse en Californie

en sont encore à des phases de prospection. Les projets de la Western Alliance [18], du Pacific Northwest Rail Corridor [19] et du Texas ${ }^{8}$ restent embryonnaires. Enfin, la ligne à grande vitesse en Floride a été abandonnée par le gouverneur Rick Scott en $2011^{9}$.

\section{Le discours de la CHSRA :} un projet avant tout rhétorique?

Le corridor californien semble le projet le plus crédible et est le plus avancé [20]. Le projet californien est mené par une agence régionale qui dépend du pouvoir de Sacramento et du gouverneur Jerry Brown. Les acteurs rencontrés sur le terrain ( $c f$. Annexe) et qui ne travaillent pas pour la CHSRA

\footnotetext{
${ }^{8}$ Seul le site internet de la Texas High Speed Rail and Transportation Corporation fournit des informations : www.thsrtc.com

${ }^{9}$ Williams T., 2011, « Florida's Governor Rejects High-Speed Rail Line, Fearing Cost to Taxpayers », New York Times (http://www.nytimes.com/2011/02/17/us/17rail.html).
}

pointent tous une évidence : les responsables du projet ne sont pas des spécialistes de la grande vitesse. Il s'agit d'un nombre très limité de personnes issues de la politique régionale et locale (comtés), généralement spécialisées dans l'aménagement des transports. L'équipe de la CHSRA ne s'est pas entourée de personnes ayant des compétences dans le domaine de la grande vitesse.

Le projet, encore très rhétorique, est avant tout développé sur le papier dans deux rapports successifs de novembre 2011 [21] et d'avril 2012 [3]. Dans la deuxième partie du rapport de 2012, intitulée The Implementation Strategy, les auteurs font très régulièrement référence à des investisseurs privés, mais ils ne sont jamais nommés et il est impossible actuellement de savoir de qui il s'agit [3]. De même, alors que la construction de la ligne doit débuter en 2013 afin que les fonds fédéraux ne soient pas perdus, la CHSRA vient tout juste d'annoncer la liste des entreprises retenues ${ }^{10}$.

\footnotetext{
${ }^{10}$ Barrow K (2013) « Land acquisition starts for California high-speed project » International Railway Journal
} 
Tableau 1 Les projets de grande vitesse aux États-Unis : un avancement contrasté

\begin{tabular}{|c|c|c|c|c|}
\hline Espace et nom du projet & Principales villes & Distance du réseau & Vitesse & Entrée en service \\
\hline Corridor Nord Est & Boston & $734 \mathrm{~km}$ & $240 \mathrm{~km} / \mathrm{h}$ & 2010 \\
\hline Acela Express & $\begin{array}{l}\text { New Haven } \\
\text { New York } \\
\text { Washington } \\
\text { D.C. } \\
\text { Philadelphie } \\
\text { Albany }\end{array}$ & & $250 \mathrm{~km} / \mathrm{h}$ & 2030 \\
\hline $\begin{array}{l}\text { Xpress West } \\
\text { (Californie du Sud et Nevada) }\end{array}$ & $\begin{array}{l}\text { Las Vegas } \\
\text { Los Angeles }\end{array}$ & $300 \mathrm{~km}$ & $240 \mathrm{~km} / \mathrm{h}$ & 2018 \\
\hline Texas & $\begin{array}{l}\text { Dallas } \\
\text { Austin } \\
\text { Houston } \\
\text { San Antonio }\end{array}$ & $780 \mathrm{~km}$ & $320 \mathrm{~km} / \mathrm{h}$ & 2020 \\
\hline Californie & $\begin{array}{l}\text { Sacramento } \\
\text { San Francisco } \\
\text { San Jose } \\
\text { Los Angeles } \\
\text { San Diego }\end{array}$ & $1200 \mathrm{~km}$ & $350 \mathrm{~km} / \mathrm{h}$ & $\begin{array}{l}2022 \\
2029\end{array}$ \\
\hline Midwest & $\begin{array}{l}\text { Chicago- } \\
\text { Detroit- } \\
\text { Cleveland } \\
\text { Chicago- } \\
\text { Minneapolis } \\
\text { Chicago-Saint } \\
\text { Louis } \\
\text { Chicago- } \\
\text { Cincinnati }\end{array}$ & $\begin{array}{l}672 \mathrm{~km} \\
707 \mathrm{~km} \\
497 \mathrm{~km} \\
454 \mathrm{~km}\end{array}$ & $350 \mathrm{~km} / \mathrm{h}$ & 2030 \\
\hline $\begin{array}{l}\text { Western Alliance } \\
\text { (Nevada, Arizona, Colorado et Utah) }\end{array}$ & $\begin{array}{l}\text { Denver } \\
\text { Salt Lake City } \\
\text { Las Vegas } \\
\text { Phoenix }\end{array}$ & Itinéraire précis non fixé & $240 \mathrm{~km} / \mathrm{h}$ & Pas de date \\
\hline Pacific Northwest Rail Corridor & $\begin{array}{l}\text { Eugene } \\
\text { Portland } \\
\text { Seattle } \\
\text { Vancouver }\end{array}$ & $605 \mathrm{~km}$ & Non fixé & Pas de date \\
\hline Floride & $\begin{array}{l}\text { Tampa } \\
\text { Orlando }\end{array}$ & $135 \mathrm{~km}$ & $270 \mathrm{~km} / \mathrm{h}$ & Rejeté en 2011 \\
\hline
\end{tabular}

Réalisé par C. Ruggeri en 2012 d'après le NEC Master Plan Working Group, le Xpress West, la Texas High Speed Rail and Transportation Corporation, la California High Speed Rail Authority, la Midwest High Speed Rail Association, la Western High Speed Rail Alliance et le Washington State Department of Transportation 
Les éléments les plus concrets développés par la CHSRA concernent le financement. Initialement prévu autour de 40 milliards de dollars, le projet atteint les 98 milliards de dollars fin 2011 [21]. En avril 2012, les coûts ont été ramenés à 68 milliards de dollars [3]. Actuellement, seules deux sources de financement sont identifiées : les fonds fédéraux issus du gouvernement fédéral de Barack Obama et les fonds fédérés issus de l'État californien. L'enjeu $\mathrm{du}$ financement $\mathrm{du}$ projet pose donc la question de la faisabilité de cette infrastructure [22]. La CHSRA dispose pour le moment d'un budget de 13 milliards de dollars dont 2,34 milliards de fonds fédéraux [12]. Ces fonds fédéraux qui doivent être utilisés avant 2018 obligent la CHSRA à commencer les travaux en $2013^{11}$. Sur le long terme, l'État fédéral n'annonce pas de nouvelle subvention pour la grande vitesse et cette absence de certitude est un enjeu financier de taille pour la CHSRA [23].

Les premiers travaux concernant une section de 130 miles (soit 208 kilomètres) du nord de Fresno jusqu'au nord de Bakersfield font débat [24] [25]. En effet, dans le contexte économique actuel et vu le grave déficit budgétaire de la Californie, de nombreux acteurs politiques (majoritairement républicains) accusent l'État californien de dépenser de l'argent qu'il n'a pas. Par ailleurs, de nombreux observateurs et acteurs se demandent s'il n'aurait pas fallu commencer par la construction de sections à partir de San Francisco ou de Los Angeles (par exemple entre Los Angeles et Bakersfield ou Los Angeles et San Diego). Ainsi, même si la ligne à grande vitesse n'était pas entièrement achevée, les sections construites seraient utiles et fréquentées, contrairement à cette section de 130 miles desservant des villes intermédiaires. Dans ce contexte, pourquoi la Californie affiche-t-elle une telle volonté politique et que cachent ce discours et cette ambition politique ?

Les arguments de la CHSRA sont en apparence simples et reposent sur les trois piliers du développement durable : économique, environnemental et social. Ces arguments sont classiques pour justifier la construction d'une ligne à grande vitesse [26].

Économiquement, le projet pourrait créer environ 160000 emplois dans le secteur de la construction selon la CHSRA [21], puis engendrer une économie indirecte liée aux activités touristiques d'hôtellerie, de restauration et de loisirs [27]. Si l'on y ajoute les 450000 emplois permanents indirects, ce chiffre (comme tous ceux communiqués par la

(http://www.railjournal.com/index.php/north-america/landacquisition-starts-for-california-high-speed-rail.html). Les entreprises retenues sont la California Backbone Builders, la California High-Speed Rail Partners, la California High-Speed Rail Ventures, Dragados/Samsung/Pulice et Tutor Perini/Zachry/Parsons.

${ }^{11}$ Les travaux devraient finalement commencer en 2014 (http://articles.latimes.com/2013/aug/11/local/la-me-bullet-delays20130812)
CHSRA) semble disproportionné et procède d'une prévision dont la méthode n'est pas expliquée [21]. David Levinson montre qu'actuellement la construction représente 577000 emplois en Californie et que ce seul projet représenterait donc un quart à un tiers des emplois de ce secteur. Il en conclut que de tels chiffres relèvent plus de la propagande que de la réalité [28].

Pour l'environnement, le train à grande vitesse est présenté par la CHSRA et les différents acteurs liés aux projets comme une alternative à l'automobile et à l'avion. La CHSRA estime que $75 \%$ des trajets actuels entre Sacramento et San Diego s'effectuent en voiture et $24 \%$ en avion [3]. Cela représente une opportunité de redistribution modale dans un État où l'automobile monopolise $97 \%$ des déplacements [3]. Le train à grande vitesse-avec un temps de trajet de 2 h $40^{12}$ entre San Francisco et Los Angeles-peut rivaliser avec les navettes aériennes qui mettent 83 minutes à relier les deux villes ${ }^{13}$ si l'on prend en compte le temps dit «perdu au sol».

Ce temps perdu au sol qui inclut les trajets de et vers les aéroports est difficile à évaluer et aucune source ne fournit de chiffres. Cependant, les éléments suivants sont listés à titre d'exemples illustratifs : le trajet en BART (Bay Area Rapid Transit) entre le centre de San Francisco et l'aéroport dure, à lui seul, 35 minutes au minimum ${ }^{14}$; l'aéroport de Los Angeles n'est pas desservi par le métro (un trajet en voiture vers les villes les plus proches, telles que Venice, peut ne prendre qu'un quart d'heure si le trafic est fluide, mais c'est une situation assez rare en journée à Los Angeles) ; les réseaux routiers et les aéroports de Californie sont saturés (selon la CHSRA [3] et les membres du SCAG (Southern California Association of Governments) ${ }^{15}$ interrogés) et il n'est pas envisageable de construire de nouvelles infrastructures routières ou aéroportuaires.

Le train à grande vitesse peut permettre de corriger des disparités sociales d'accessibilité. En effet, ce train desservirait des villes où les liaisons aériennes et les services de bus sont limités (comme à Merced, Modesto ou Stockton). Enfin, la CHSRA met en avant une intermodalité accrue puisque le train à grande vitesse serait connecté aux réseaux Amtrak, mais aussi aux réseaux Caltrain et BART dans la baie de San Francisco et Metrolink dans l'aire urbaine de Los Angeles; sans oublier de possibles gares aux aéroports de San Francisco, de Burbank voire d'Ontario.

\footnotetext{
12 Selon le rapport 2012 de la CHSRA [3]. Ce temps de gare à gare n'inclut pas le temps de transport de/vers la gare.

${ }^{13}$ Temps moyen des vols réguliers entre Los Angeles et San Francisco en janvier 2012 selon les bases de données d'OAG.

${ }^{14}$ Selon une estimation faite par l'auteur sur le site du BART (www.bart.gov) à partir des horaires de journée.

${ }^{15}$ Le SCAG est la MPO de la région urbaine de Los Angeles. En 2012, un entretien a été réalisé avec Stephen G. Fox, responsable du projet de la grande vitesse pour le SCAG.
} 
Ce discours argumenté, très détaillé et parfois pertinent est défendu par la CHSRA dans ses deux rapports successifs, sur son site internet et dans de multiples réunions publiques, mais il semble avant tout rhétorique et s'inscrit dans un discours et dans un mythe plus large sur la «Californie du futur »[3] [21]. En effet, la CHSRA développe l'idée d'un train innovant qui va placer la Californie dans le club assez restreint des territoires desservis par un train à grande vitesse. Le discours sur la capacité d'innovation, sur l'aptitude californienne à être un laboratoire et à créer avant les autres États américains, trouve un écho fort dans le train à grande vitesse. La plupart des membres de la CHSRA, du SCAG, du MTC (Metropolitan Transportation Commission) ${ }^{16}$, du Metro Los Angeles ou des responsables politiques locaux (Anaheim) pensent que le projet ne pouvait voir le jour qu'en Californie et que la Californie donne l'exemple en matière d'innovation afin d'inspirer d'autres États américains sur la voie de la grande vitesse. Cette croyance relève d'une conception particulière de l'image, de l'identité et du territoire californiens.

\section{Du discours à la réalité : un territoire face à la grande vitesse}

Au-delà d'un discours rhétorique, que les années infirmeront ou confirmeront, la future ligne à grande vitesse californienne dessine des lignes de tension ou de convergence qui révèlent la multiplicité et la complexité des territoires, des échelles, des réseaux et des acteurs qui constituent ou gravitent autour de ce projet.

\section{Le train à grande vitesse, vecteur de centralisation ou de décentralisation?}

Ce projet, piloté par une agence régionale dépendante $\mathrm{du}$ pouvoir de Sacramento, pose la question du rôle et de l'attitude du pouvoir central californien au delà de la construction d'un discours. Ce projet qui (selon la CHSRA [3]) irrigue les trois quarts du territoire californien et la plupart des grandes agglomérations de l'État, est-il imposé par un pouvoir central californien qui se veut fort et ambitieux dans un pays où ce type de politique d'aménagement est peu pratiqué ?

Il est possible d'envisager cette stratégie d'aménagement de la ligne à grande vitesse comme une revendication de centralisation politique puisqu'elle atteste d'une affirmation $\mathrm{du}$ pouvoir californien, mais également comme une politique de décentralisation par les effets de désenclavement qu'elle pourrait avoir sur certains

\footnotetext{
${ }^{16}$ La MTC est la MPO des comtés de la Baie de San Francisco.
}

territoires. Le territoire californien est déjà desservi par un réseau de transport dense, soutenu par un réseau urbain dominé par deux ensembles puissants au Nord (San Francisco/San Jose/Sacramento) et au Sud (Los Angeles/San Diego). Mais, selon les échelles, les réseaux de transport s'avèrent partiels et lacunaires.

À l'échelle régionale, les réseaux autoroutiers et aériens sont certes denses, mais saturés. Le réseau ferroviaire est, quant à lui, très lacunaire. Neuf heures sont nécessaires pour relier Los Angeles à San Francisco en alternant un bus, un train puis un bus ou le BART. À l'échelle infrarégionale les lacunes d'infrastructures sont plus évidentes, notamment les dessertes ferroviaires et aériennes inexistantes dans de nombreuses agglomérations. À l'échelle urbaine, les réseaux sont très denses mais complexes à appréhender, étant donné la multitude d'opérateurs (les politiques de transports sont gérées par les MPO). Pour les transports publics, la coordination peut sembler inextricable, notamment dans la Baie de San Francisco où 28 opérateurs desservent différents territoires à différentes échelles. Los Angeles et San Diego disposent de structures plus unifiées-autour du SCAG et du SANDAG (San Diego Association of Governments), les deux MPO - et d'un opérateur unique. La CHSRA présente donc le projet de ligne à grande vitesse comme une opportunité d'articulation des réseaux multiscalaires et multiactoriels par un réseau de strate supérieure [3] [21].

En effet, ce train à grande vitesse propose de développer une puissante intermodalité avec des connexions aux réseaux ferroviaires existants et la desserte de nombreuses infrastructures aéroportuaires (San Francisco, Burbank, Ontario). Le dernier rapport de la CHSRA prévoit même un blended system qui correspond à une articulation des réseaux urbains locaux avec la ligne à grande vitesse, afin que les réseaux locaux prennent le relais du train à grande vitesse le temps que la construction de la ligne soit complète [3]. La CHSRA défend également son projet comme un moyen de désenclaver des territoires délaissés par les liaisons routières en bus, ferroviaires et aériennes.

Le comblement de ces lacunes territoriales de transport entraînera très probablement une transformation ou une adaptation des pratiques de mobilité des Californiens susceptibles de voyager en train à grande vitesse. Les Californiens sont en effet habitués à se déplacer en voiture et en avion et, lorsqu'ils se déplacent en avion, ils réalisent majoritairement leurs trajets de et vers l'aéroport en voiture. Cela a provoqué le développement d'immenses zones à perte de vue autour des aéroports où se regroupent les loueurs de voiture, notamment à Los Angeles. Le discours de la CHSRA aborde la question de ces pratiques de mobilité avec l'idée d'une redistribution des flux aériens afin de répondre à la saturation des aéroports de Los Angeles et de San Francisco. Les compagnies aériennes pourraient envisager 
de créer des liaisons aériennes régionales et nationales vers les aéroports autour de Los Angeles (Burbank au nord, Ontario à l'est) et de San Francisco (San Jose au sud) reliés aux centres de ces deux villes en une demi-heure par le train à grande vitesse [3] ( $c f$. Fig. 1) et donc sans obliger les usagers à louer une voiture. Cette conception de la redistribution modale qui place le train à grande vitesse en support de l'activité aérienne est paradoxale vis-à-vis des arguments environnementaux avancés par la CHSRA. Au-delà de cette contradiction, une concurrence risque surtout d'apparaître entre le transport aérien et le train à grande vitesse [23].

Le choix de Burbank n'est toutefois pas encore arrêté puisque deux arrêts potentiels sont encore à l'étude sur le tracé Palmdale-Los Angeles [29]. Actuellement, l'aéroport Bob-Hope de Burbank qui assure essentiellement des liaisons régionales et qui est l'un des mieux placés pour être connecté à la ligne à grande vitesse est uniquement connecté au réseau Metrolink qui dessert la Californie $\mathrm{du}$ Sud. Ces infrastructures assez sommaires (pas de parking réservé aux usagers du Metrolink et les piétons doivent traverser une route assez fréquentée puis le parking pour rejoindre l'aéroport à pied) peuvent prétendre à une meilleure intégration au réseau de transport sud californien, voire californien, à condition de la mise en place de mesures d'accompagnement et d'aménagement à la hauteur. Le train à grande vitesse n'a pas la capacité de transformer l'aéroport de Burbank en hub national, ni même régional, mais une politique pertinente et réfléchie d'aménagement en amont permettrait probablement à celui-ci de s'affirmer dans le réseau de transport californien. Par ailleurs, au-delà de la captation de mobilités touristiques, Burbank peut faire valoir des atouts touristiques typiquement californiens comme les studios Warner Bros et Universal qui se visitent. Si Burbank obtient la gare au détriment de San Fernando/Sylmar, des travaux d'aménagements d'envergure restent à envisager et à entreprendre.

Ontario, dans l'aire urbaine de Los Angeles, dispose quant à elle de deux gares Metrolink et d'un aéroport [30]. Les deux gares ont été aménagées, sont connectées aux réseaux de bus de la ville et disposent de grands parkings permettant aux usagers de garer leur voiture afin de se rendre en train dans le centre de Los Angeles par exemple. Ces deux villes, qui sont face à la même opportunité de ligne à grande vitesse ne disposent pas des mêmes aménagements initiaux et seule les ambitions municipale et locale leur permettront de réussir (ou non) leur connexion au réseau à grande vitesse.

Considérer l'arrivée d'une ligne à grande vitesse comme une opportunité d'aménagement du territoire est courant. On a tendance à voir le train à grande vitesse comme un levier économique et territorial, mais l'effet d'entraînement sur les villes n'existe que si le dynamisme économique et la volonté publique locale préexistent [31]. L'étude de l'attitude des municipalités, mais également des comtés, révèle une dichotomie entre un robuste ancrage local du projet et un rejet tout aussi fort.

\section{Entre ancrage local et rejet : les territoires locaux face à la desserte à grande vitesse}

La CHSRA a exigé des dix villes principales susceptibles d'accueillir une gare la construction de nouvelles infrastructures en centre-ville (downtown) afin de préparer l'arrivée du futur train à grande vitesse. Sans action d'accompagnement en amont, les acteurs territoriaux ne peuvent pas espérer de «miracle » de la grande vitesse [32].

D'un point de vue politique, les stratégies d'acteurs existent, mais elles n'attestent pas pour autant d'une adhésion au projet de grande vitesse qui peut revêtir plusieurs formes : individuelle relevant d'un acteur privé ou public ou collective permettant une coordination de plusieurs acteurs autour d'un même projet. Une action isolée est rarement pertinente et les acteurs locaux doivent prendre conscience de la nécessité de construire un projet cohérent d'accompagnement de la nouvelle desserte ferroviaire à l'échelle du territoire [1].

Les positions et stratégies locales des villes californiennes diffèrent : certaines acceptent et soutiennent pleinement le projet depuis le début et se l'approprient en termes d'aménagements (Palmdale, Anaheim, San Francisco); d'autres l'acceptent mais leur appropriation en est encore à un stade initial (Bakersfield, Los Angeles) ; d'autres encore profitent, ou pourraient profiter, du projet pour redynamiser leurs politiques locales de transport (Sylmar/San Fernando, Burbank, Ontario). Aucune municipalité n'est opposée au projet lorsqu'une gare est prévue, en revanche leur appropriation est toute autre lorsque le train à grande vitesse ne doit que traverser le territoire. Dans la ville de Santa Clarita, le maire, une majorité du conseil municipal, la population et les acteurs économiques s'opposent vivement au tracé de la ligne entre Palmdale et Los Angeles qui doit passer à quelques kilomètres de la ville.

Par ailleurs, alors que la CHSRA pensait éviter les oppositions locales en commençant à construire dans les espaces peu urbanisés du nord du tracé entre Fresno et Bakersfield, une association d'agriculteurs et de riverains du comté de Kings (la Citizen for California High Speed Rail Accountability) mène l'opposition et a déposé plainte contre la CHSRA. Plusieurs associations de défense de l'environnement commencent également à déposer plainte-d'autant plus que Jerry Brown tente d'obtenir des passse-droits 


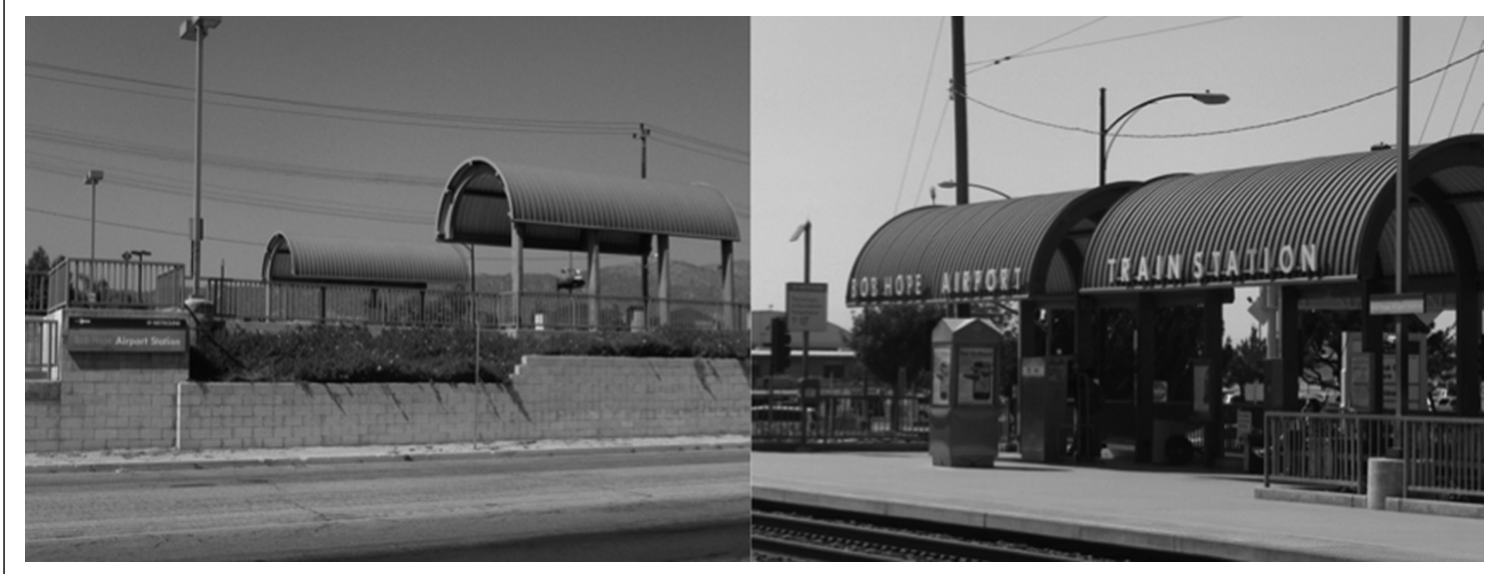

Fig. 2 La gare Metrolink Bob Hope à Burbank (cliché C. Ruggeri, 2012)

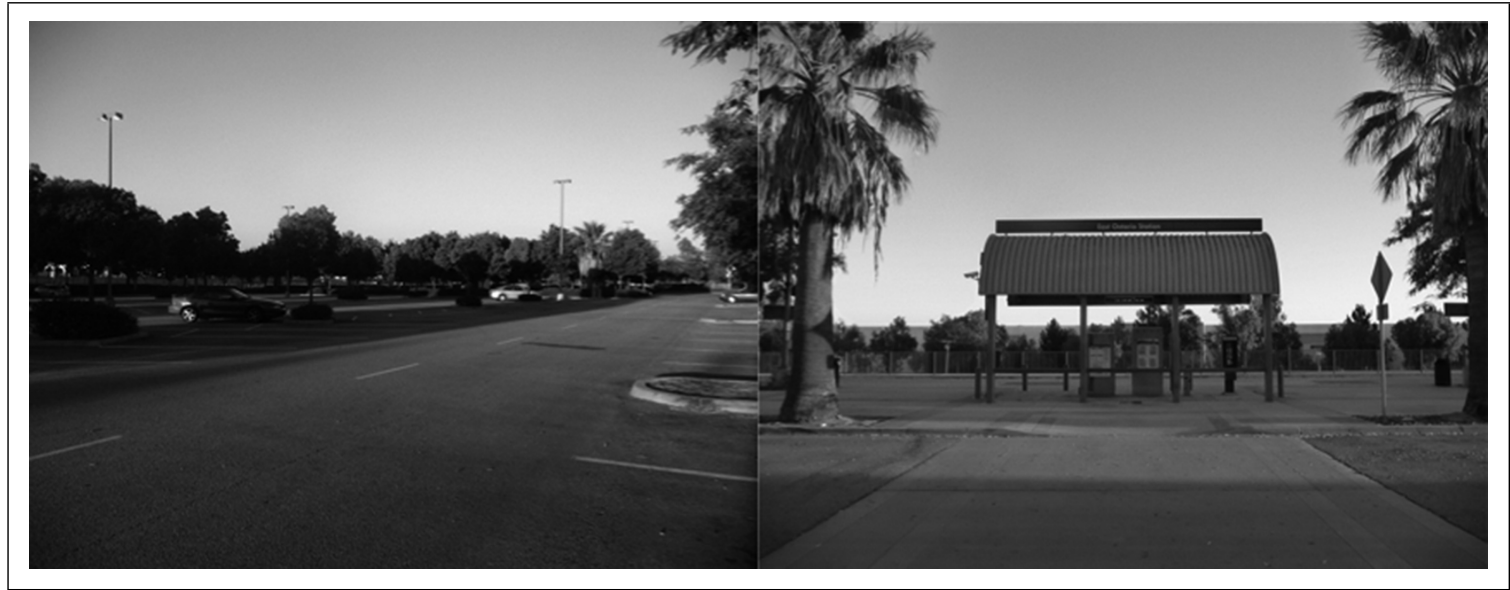

Fig. 3 La gare Metrolink d'East Ontario (cliché C. Ruggeri, 2012)

environnementaux pour la construction de la ligne à grande vitesse $^{17}$

Même dans les villes desservies, certains acteurs doutent de la pertinence du projet. C'est le cas à Anaheim, où la ligne à grande vitesse est un véritable sujet de débat entre d'une part la municipalité, la chambre de commerce et le comté favorables au projet et d'autre part l'autorité des transports du comté, l'OCTA (Orange County Transportation Authority) qui désapprouve publiquement le projet, estimant son coût exorbitant. L'OCTA développe également l'idée qu'il serait plus pertinent d'utiliser les fonds prévus pour le projet à grande vitesse pour améliorer le réseau ferroviaire californien vieillissant ${ }^{18}$. Cet argument

\footnotetext{
${ }^{17}$ Vartabedian R (2012) « Head of bullet train seeks eco-exemptions », Los Angeles Times (http://articles.latimes.com/2012/may/16/local/lame-bullet-hearing-20120516).

${ }^{18} \mathrm{~T}$ (2012) « OCTA unfriendly to High-Speed Rail », Voice of OC (http://www.voiceofoc.org/countywide/this_just_in/article_588fbb429ea2-11e1-9a1c-0019bb2963f4.html).
}

est courant chez les opposants à la grande vitesse aux États-Unis qui défendent des investissements moins coûteux et moins risqués [27]. Les défenseurs du projet avancent avant tout des impacts économiques favorables au comté d'Orange, comme l'estimation de la création de 23000 emplois d'ici 2030. D'un point de vue économique toujours, le futur train à grande vitesse permettrait de faciliter l'accessibilité du comté et en particulier d'Anaheim qui a de nombreuses aménités touristiques à offrir. Le parc d'attraction de Disneyland est en effet à quelques kilomètres seulement de la future gare ARTIC (Anaheim Regional Transportation Intermodal Center) qui accueillera tous les réseaux de transport irriguant le comté et la ville (train à grande vitesse, Metrolink, bus de l'OCTA, navettes du Anaheim Resort Transit). Le comté reprend également les arguments traditionnels de redistribution modale des mobilités comme celui de la distance et du trafic pour rejoindre l'aéroport de Los Angeles, de la saturation de l'aéroport John-Wayne et des impacts environnementaux [27]. 


\begin{tabular}{|c|c|c|c|}
\hline Soutien fort & Soutien moyen & Soutien faible & Rejet \\
\hline Anaheim & Burbank & Bakersfield & Comté de Kings \\
\hline Comté d'Orange & Fresno & Comté de Kern & OCTA \\
\hline $\begin{array}{l}\text { Chambre de } \\
\text { commerce }\end{array}$ & Merced & Los Angeles & Santa Clarita \\
\hline d'Orange & Sacramento & San Fernando/Sylmar & \\
\hline Fresno & & & \\
\hline Metro LA & & & \\
\hline MTC & & & \\
\hline Ontario & & & \\
\hline Palmdale & & & \\
\hline San Diego & & & \\
\hline San Francisco & & & \\
\hline San Jose & & & \\
\hline SCAG & & & \\
\hline TransForm & & & \\
\hline
\end{tabular}

Ces éléments posent la question de l'appropriation d'une infrastructure collective dans un territoire tourné vers des infrastructures de transport individuelles [23]. L'étude et la rencontre des différents acteurs impliqués ou concernés ${ }^{19}$ par le projet ont déjà permis de cerner des profils et des lignes de partage dans la région de Los Angeles. Cette appropriation de la future infrastructure selon les acteurs (municipalités, comtés et MPO) peut être à la fois symbolique (acceptation) et/ou pratique (aménagements).

Le soutien fort se traduit par des aménagements d'envergure et par des acteurs qui défendent publiquement le projet ; le soutien dit moyen implique des aménagements importants qui peuvent être contestés par d'autres acteurs (de la société civile notamment); le soutien faible concerne les acteurs qui, pour le moment, n'affichent pas un engagement fort dans le projet et des territoires dont les aménagements prévus demeurent timides ou purement techniques et obligatoires pour accueillir les futurs trains; le rejet concerne les acteurs qui s'opposent au projet.

Les opposants au projet, à commencer par l'OCTA et un certain nombre de municipalités comme Santa Clarita, dénoncent une utilisation ni raisonnée ni raisonnable des fonds publics. Ils craignent avant tout une augmentation des impôts. Ce rejet des grandes infrastructures publiques est assez classique aux États-Unis. Depuis le réseau des Interstates, lancé en 1956, aucun autre grand projet de

\footnotetext{
${ }^{19}$ Voir l'annexe « Liste des acteurs rencontrés cités dans l'article ».
}

${ }^{20}$ Les villes citées sont représentées par leur municipalité. construction d'infrastructure n'a été lancé en Californie. Des acteurs (comme les responsables du SCAG, du Los Angeles Metro ou d'ARTIC) expliquent que les Américains n'ont pas la culture de l'équipement et de l'infrastructure publique financée presque entièrement par des fonds publics. De plus, ils signalent l'absence de culture ferroviaire contemporaine (au sens d'habitudes et de pratiques mobilitaires). Les Californiens prennent peu le train et il est donc difficile de défendre la construction d'une infrastructure qui doit faire ses preuves et qui n'est pas inscrite dans les mentalités [4]. Tous ces éléments posent la question de l'acceptation du projet, mais aussi des motivations politiques qui sous-tendent le débat.

\section{Le remodelage de la hiérarchie urbaine}

La position des acteurs territoriaux peut évoluer puisque le projet de ligne à grande vitesse est développé depuis neuf ans $^{21}$. Ainsi, certaines villes expliquent attendre de voir comment il évolue pour prendre position et dévoiler leurs projets d'aménagement relatifs à la grande vitesse ${ }^{22}$. À l'inverse, d'autres villes ont très tôt envisagé d'ambitieux projets d'aménagement

\footnotetext{
${ }^{21}$ Le projet actuel est en effet développé depuis 2004, même si l'Intercity High-Speed Rail Commission existe depuis 1993.

${ }^{22}$ C'est le cas des villes du corridor sud, entre Los Angeles et San Diego, dont Los Angeles qui a commencé les études préliminaires de réaménagement d'Union Station en mai 2013.
} 
du territoire en vue de l'arrivée de la grande vitesse et sont revenus sur ces projets - pour des raisons politiques ou financières, voire les deux le plus souvent.

C'est le cas de la ville de Bakersfield. Lorsque le projet avait été dévoilé, cette ville avait projeté une gare exurbanisée dans un nouveau quartier au sud, une sorte de ville nouvelle à l'américaine. Ce nouveau quartier aurait été un quartier purement résidentiel, destiné à accueillir les commuters qui travaillent à Los Angeles. La ville de Bakersfield est revenue assez rapidement sur ce projet en proposant plutôt une gare en centre-ville, à l'image de Fresno [24]. Toutefois, cette gare et ce tracé sont contestés par le conseil municipal et le comté de Kern depuis juillet 2012. Ils préféreraient un tracé plus au sud, à l'extérieur de la ville. On en reviendrait donc à une gare exurbanisée au sud, connectée à l'autoroute 99.

Cet exemple démontre la difficulté des acteurs locaux à défendre un projet commun, pertinent et stable [1]. Pourtant, ces territoires, et en particulier les villes, sont les principaux bénéficiaires de la ligne à grande vitesse [33]. Les villes secondaires du réseau urbain californien (Merced, Fresno, Bakersfield, Stockton, Hanford, Modesto, Palmdale, Gilroy) sont les plus concernées par les lacunes des réseaux de transport. Leur accessibilité limitée se réduit souvent à la voiture, voire au bus avec des trajets très longs. L'étude des projets d'aménagements urbains et de transports dans ces villes permet de repenser la hiérarchie urbaine californienne [32]. Quel statut ces villes, pour la plupart très mal desservies en transport, peuvent-elles espérer obtenir si la grande vitesse engendre un remodelage du réseau urbain californien ?

La préférence de la ville de Bakersfield pour une gare exurbanisée marque le retour à un constat initial : le train à grande vitesse renforcerait les pôles urbains californiens (San Francisco, Los Angeles, San Diego) et les bénéfices pour les villes intermédiaires seraient faibles. De fait, la ville avance divers arguments contre le projet de gare centrale. Selon la municipalité et le comté, cela ne ferait qu'accroître la congestion dans le centre. Avec une gare TGV dans le centre, il faudrait ajouter aux mobilités quotidiennes pendulaires les déplacements des personnes se rendant à la gare pour aller travailler dans un autre centre urbain. Ainsi, selon la municipalité, puisqu'une part importante de la population vit dans les espaces périurbains, autant aménager la gare dans ces espaces afin de limiter les mobilités automobiles vers le centre de Bakersfield. Les habitants de Bakersfield ayant besoin de se rendre à Palmdale ou à Los Angeles pour leur travail disposeraient ainsi d'une gare à proximité.

À l'inverse, la ville de Palmdale soutient le projet de grande vitesse depuis le début. Ainsi la CHSRA, qui avait un temps envisagé une section Bakersfield-Los Angeles sans arrêt à Palmdale, a revu ses plans [24] [29]. La ville de Palmdale est consciente que la prééminence de Los Angeles dans le sud de la Californie ne va pas s'amenuiser avec la grande vitesse, mais cette dernière ne doit pas pour autant être considérée comme un accélérateur du décrochage des villes secondaires face aux pôles urbains californiens. Pour des villes comme Palmdale, Burbank, Ontario ou Riverside, la ligne à grande vitesse présente une opportunité de mise en réseau accrue. Pour ces villes, accessibles de Los Angeles par autoroute et par le Metrolink, la grande vitesse offre une infrastructure nouvelle et rapide densifiant le réseau de transport du sud californien et l'étendant jusqu'à San Diego.

Le soutien très fort du projet par des villes comme Palmdale laisse penser qu'elles attendent beaucoup de la nouvelle infrastructure. Il est difficile, voire impossible, d'anticiper si ces espoirs se concrétiseront ou aboutiront [2] [31], De fait, ces espoirs dépendent d'éléments encore inconnus tels que la fréquence et le prix des billets du train à grande vitesse.

\section{De l'ancrage territorial à la restructuration urbaine : entre paradoxes et mutations}

L'appropriation de cette infrastructure à grande vitesse ne relève pas uniquement de l'adhésion politique des acteurs locaux. Ce train dévoile d'ores et déjà leur capacité ou leur volonté à se saisir de ce projet pour restructurer les territoires métropolitains et centraux [1] [32] [34].

\section{Un paradoxe urbain : un train urbain pour un territoire périurbain}

Le projet de ligne à grande vitesse révèle en effet une forme de paradoxe urbain propre aux villes américaines. Les activités et la population se concentrent peu dans les centres, surtout à Los Angeles et à San Diego. Même San Francisco, longtemps présentée comme un contre-modèle urbain américain, doit aujourd'hui son développement économique à la Silicon Valley, au sud de la Baie. San Jose est devenue la ville la plus peuplée de la Baie de San Francisco (avec plus de 900000 habitants en 2006) et la population augmente fortement dans les comtés de l'est et du sud de la Baie, notamment autour des corridors autoroutiers de l'interstate 580 et de l'interstate 680. Entre 1995 et 2005, la population de Dublin ${ }^{23}$ a augmenté de $52 \%$, contre seulement $6 \%$ à Oakland [35].

De fait, le projet de la CHSRA qui est de desservir les villes en leur centre grâce à de nouvelles gares, peut

\footnotetext{
${ }^{23}$ Dublin est une ville de l'est de la Baie de San Francisco (voir Fig. 5).
} 


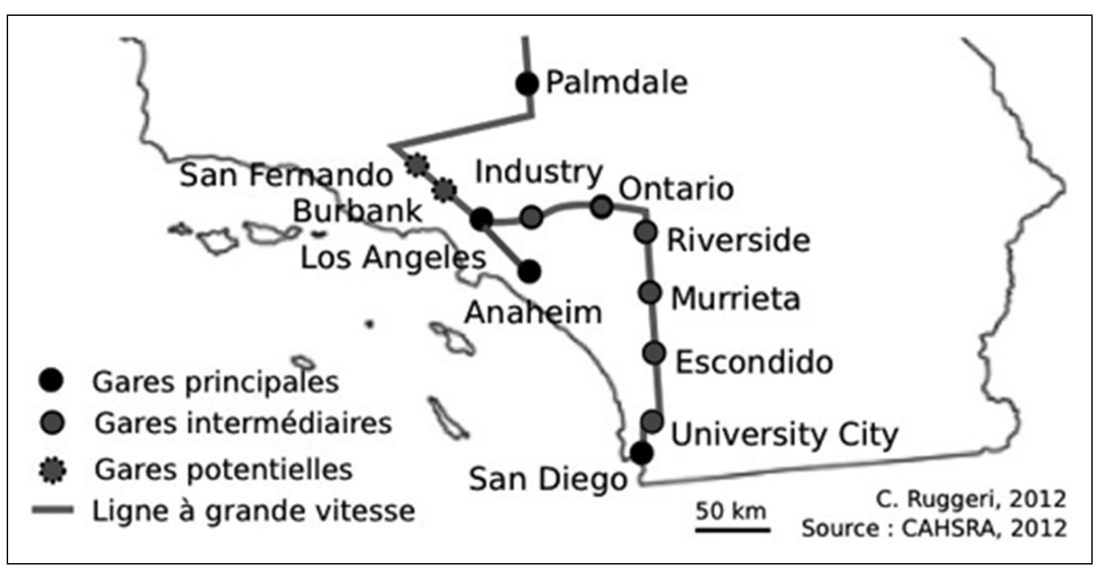

Fig. 4 Le projet de réseau sud californien de la grande vitesse

sembler paradoxal spatialement si la population et les activités se concentrent en périphérie urbaine. Si l'on prend l'exemple de downtown Los Angeles, les faits sont sans appel : on compte environ 40000 résidents dans downtown, alors que la ville en totalise 3 millions et l'aire urbaine entre 15 et 18 millions [36]. Les activités-que ce soit le cinéma et l'audiovisuel à Burbank ou le tourisme entre les plages de Santa Monica et de Venice et Disneyland à Anaheim - sont également excentrées. Downtown concentre avant tout des activités tertiaires, telles que des services de banque, de finance, d'assurance ou d'immobilier, ainsi que de très nombreux cabinets d'avocats et d'expertises. On y trouve également tous les services municipaux et judiciaires de la ville et les institutions du comté. De fait, le train à grande vitesse, qui arriverait en gare d'Union Station au nord de downtown, pourrait pleinement profiter aux mobilités professionnelles entre Sacramento, San Francisco, Los Angeles et San Diego.

Mais en termes de mobilités touristiques, on peut se demander si la localisation de la gare est pertinente. L'aéroport est ainsi bien plus proche de Venice et de Santa Monica. Par ailleurs, implanter la gare en plein downtown impliquerait d'améliorer les lignes Metrolink/Metro dans l'agglomération. Les membres du SCAG et de Metro Los Angeles ne craignent pas le manque de pertinence de la gare puisque, selon eux, downtown est en mutation. Ainsi, ils constatent une augmentation de la population résidente ${ }^{24}$, notamment parce que le foncier et les loyers sont devenus inabordables à Santa Monica et à Venice. Cette augmentation est confirmée par les chiffres du US Census Bureau et du Los Angeles Department of City Planning puisque downtown Los Angeles est passé d'une population

\footnotetext{
24 Tout en ne donnant aucun chiffre relatif à cette augmentation. Selon eux, cela se constate par le dynamisme du marché immobilier.
}

de 27800 habitants en 2000 à 34800 habitants en $2008^{25}$. Pourtant, les opérations de restructuration urbaine du nord de downtown restent timides.

Enfin, la CHSRA a pensé le réseau sud du train à grande vitesse de manière multipolaire afin de répondre à l'étalement de la population et des activités dans l'aire urbaine de Los Angeles, mais également de celle de San Francisco. Par exemple, dans l'aire urbaine de Los Angeles (Fig. 4), quatre gares et une section indépendante du tracé en direction d'Anaheim sont prévues. La ville s'est mobilisée pour confirmer son inscription dans le futur réseau à grande vitesse. En effet, dans le rapport de 2011, la section Los Angeles-Anaheim (déconnectée du reste de la ligne à grande vitesse) avait disparu [21]. Le comté, la commune et la chambre de commerce se sont alors battus pour récupérer la ligne et la gare. Ainsi, la section Los Angeles-Anaheim est réapparue dans le rapport de 2012 [3]. Le parc d'attraction de Disneyland serait ainsi accessible en train à grande vitesse en moins de 25 minutes depuis le centre de Los Angeles. Cette portion du tracé est purement vouée aux mobilités touristiques et de loisirs.

Dans la Baie de San Francisco, on remarque la même multipolarité du tracé. Le futur train à grande vitesse desservirait en effet les trois principaux pôles d'activité de la Baie avec une gare à San Jose (aux portes de la Silicon Valley) une autre à l'aéroport de San Francisco et une dernière dans le centre de San Francisco [37]. Comme dans l'aire urbaine de Los Angeles, la ligne à grande vitesse viendrait se connecter et se surimposer à un réseau de transport déjà organisé et dense.

Caltrain dessert en effet déjà le sud de la Baie par ce couloir ouest, mais le BART est, pour le moment,

\footnotetext{
${ }^{25}$ Ces chiffres sont entre autres disponibles sur le site du Los Angeles Times qui a développé le projet Mapping LA avec le US Census Bureau et la municipalité de Los Angeles (projects.latimes.com/mappingla/neighborhoods).
} 


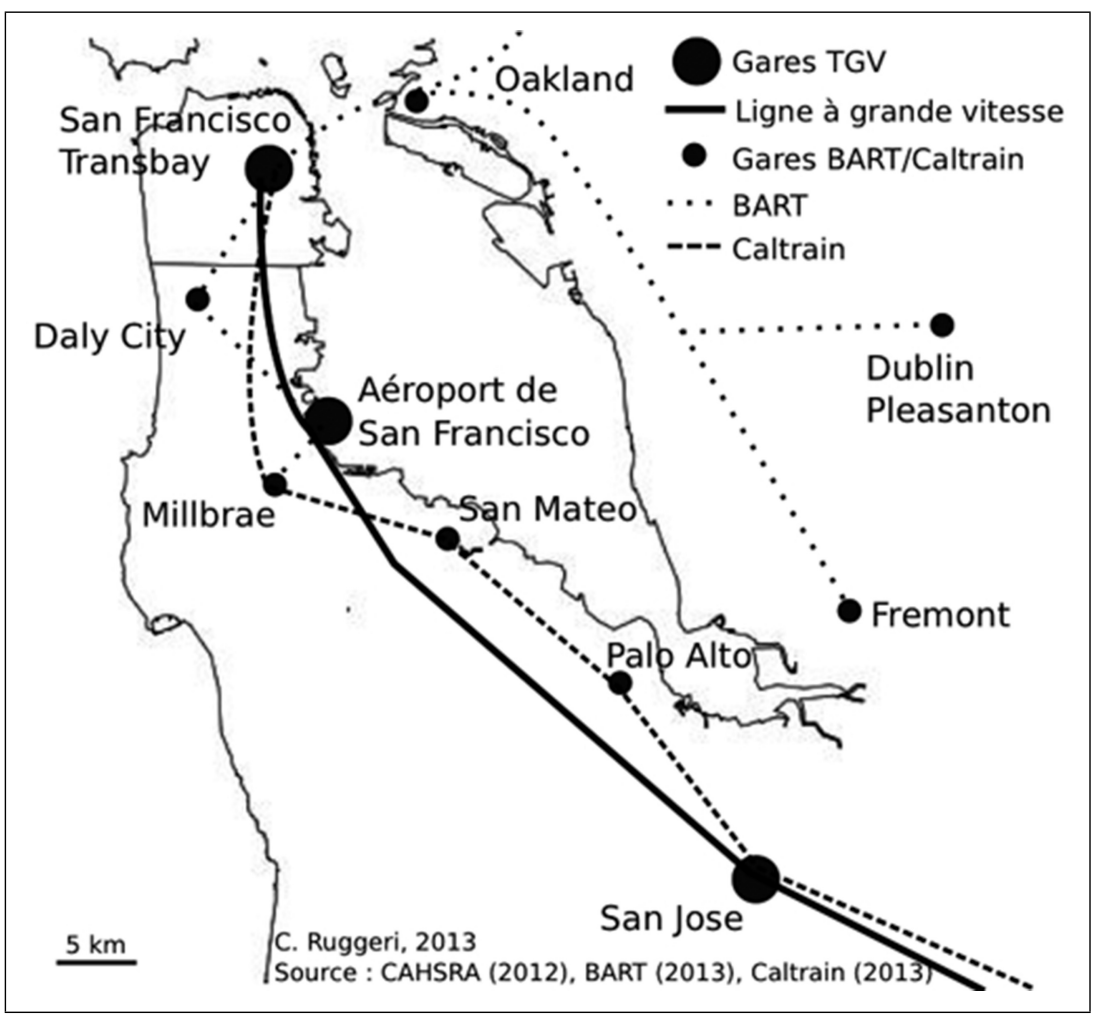

Fig. 5 Ligne à grande vitesse et réseaux ferrés dans la Baie de San Francisco

plutôt tourné vers l'est de la Baie avec l'aéroport comme station la plus au sud. Le projet d'extension du BART prévoit un aménagement du réseau jusqu'à San Jose (mais par l'est a priori) et la gare de Diridon deviendrait un pôle intermodal redistribuant les flux vers toute la Silicon Valley.

Toutefois, ces deux constructions multipolaires du réseau posent la question de la préservation de la grande vitesse. Il semble impossible de penser des trains circulant à grande vitesse entre des gares aussi proches. Ces réseaux métropolitains du train à grande vitesse sont plutôt pensés comme des réseaux de transport urbain, assurant des mobilités quotidiennes.

\section{Une ambition de restructuration urbaine}

Les gares sont des éléments architecturaux peu valorisés dans les villes californiennes ; San Francisco ne dispose ainsi pas d'une gare centrale pour les trains Amtrak (qui arrivent en gare d'Emeryville, à proximité d'Oakland). Mais avec le train à grande vitesse, chaque ville desservie doit s'équiper d'une gare nouvelle ou réhabiliter sa gare actuelle. Cela peut donc engendrer une requalification urbaine pour ces quartiers, souvent délaissés et peu fréquentés. Ces espaces pourraient alors devenir des lieux urbains centraux, redynamisés et identifiables par les habitants et par les visiteurs. Ces créations permettraient de repenser l'accessibilité centrale des villes californiennes, mais également leur identité et leur image par le prisme du train à grande vitesse et du tourisme [32].

La ville de San Francisco ne dispose que d'une gare Caltrain à l'est du quartier de South of Market (SOMA) et d'une gare Amtrak à Emeryville à l'est de la Baie pour les liaisons régionales et nationales. San Francisco, en vue d'accueillir le train à grande vitesse, a développé le projet du Transbay Transit Center ${ }^{26}$, un nouveau terminal connectant onze réseaux de transport, deux modes et quatre échelles ${ }^{27}$. Les onze opérateurs ou réseaux qui ont déjà accepté de participer au projet sont : AC Transit (réseau de bus d'Oakland et Berkeley), le BART, Caltrain, Golden Gate Transit (réseau de bus entre San Francisco et les villes au nord du pont du Golden Gate), Greyhound, Muni (réseau municipal de San Francisco), SamTrans (comté de San Mateo), WestCAT Lynx (comté de Contra Costa), Paratransit (est de la Baie), Amtrak et la CHSRA. Le terminal accueillerait un terminal de bus en surface et un terminal ferré en sous-sol. Le Muni avait pensé étendre ses lignes de tramway et de métro vers le terminal, mais a pour le moment renoncé au projet par manque de fonds.

\footnotetext{
${ }^{26}$ Voir le site internet du projet : transbaycenter.org

${ }^{27}$ L'échelle nationale, régionale, locale et san franciscaine.
} 


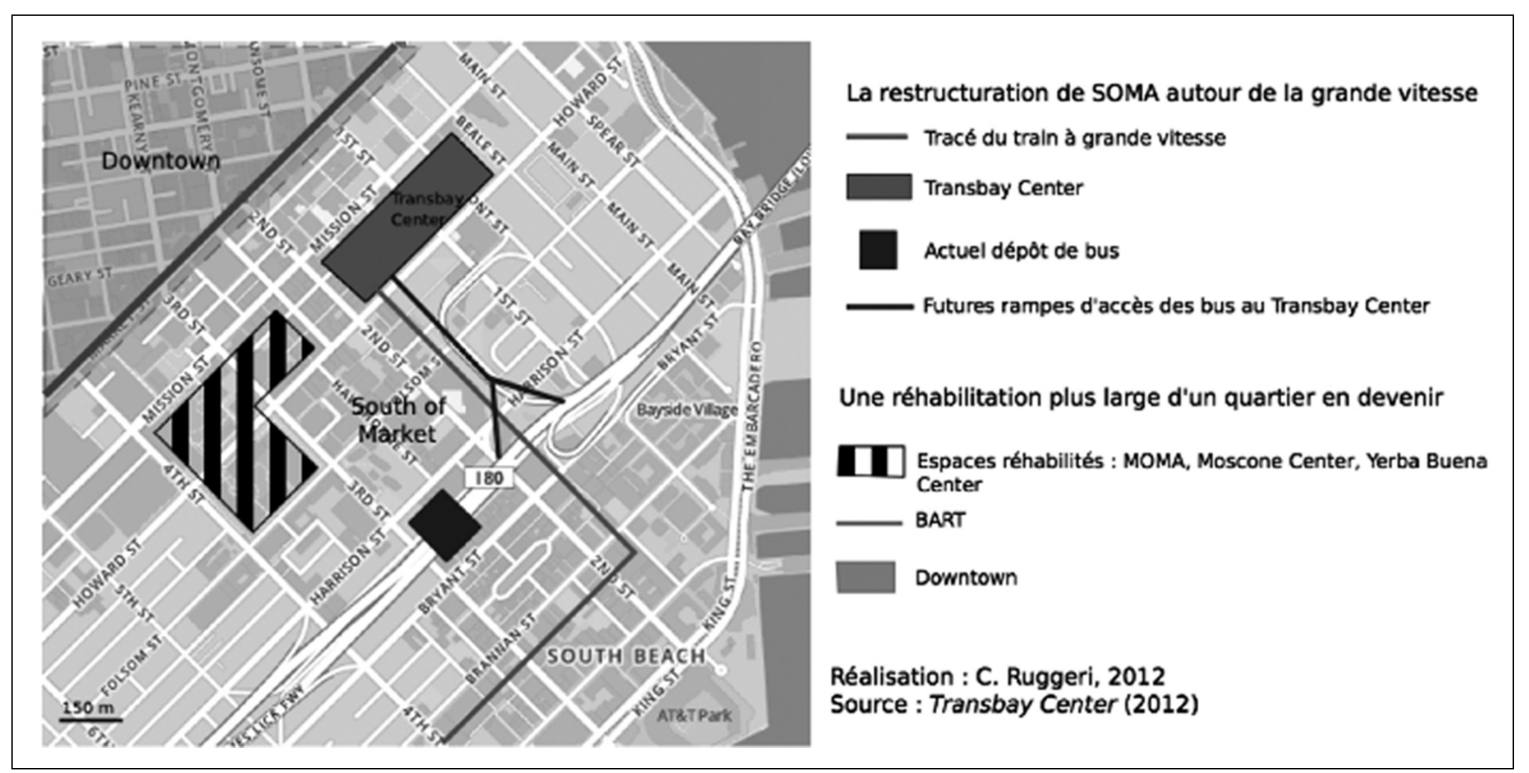

Fig. 6 Le Transbay Transit Center de San Francisco

L'intermodalité qui est donc prévue aurait pu être encore plus complète et diversifiée avec la participation du principal opérateur de transport de la ville, le Muni.

La première étape du projet est la destruction de l'actuel terminal. Un terminal de bus de remplacement a donc été installé, mais il ne s'agit en fait que d'un terrain aménagé avec des arrêts de bus. La deuxième étape, plus ambitieuse, est la construction d'une extension de la voie ferrée de Caltrain (dont le terminus est actuellement à la gare de King et Fourth Street). Cette extension se ferait en même temps que la construction de la voie à grande vitesse et le Transbay Transit Center deviendrait donc la gare à grande vitesse de San Francisco.

Enfin, une fois le nouveau terminal construit, la ville a l'intention de profiter de cette nouvelle infrastructure pour développer un tout nouveau quartier avec des logements, des bureaux, un parc et des magasins. À terme ce terminal intermodal verrait transiter 100000 personnes par jour et 45 millions de passagers par an. Ce terminal doit voir le jour dans le quartier de SOMA, un quartier qui est en plein redéveloppement et réhabilitation depuis une vingtaine d'années. Cet ancien quartier industriel, longtemps laissé à l'abandon d'un point de vue urbanistique, bénéficie d'opérations de réhabilitation et est en voie de gentrification. Il accueille aussi de nouveaux établissements depuis une dizaine d'années, tels que le Musée d'Art Moderne (MOMA), le Yerba Buena Center et le Moscone Center (centre de convention). L'évidente attractivité touristique du quartier ne cesse de se confirmer. Les immeubles de bureaux, les lofts et les appartements de standing s'y développent également, notamment à l'est, à proximité du parc de baseball AT\&T. Enfin, l'université de San
Francisco (UCSF) a ouvert un second campus au sud de South Beach, offrant notamment des logements étudiants qui manquaient sur le campus historique à proximité du Golden Gate Park. La ligne à grande vitesse a donc initié un projet d'infrastructure de transport de grande envergure qui permet à San Francisco de se doter d'une gare multimodale moderne dans un quartier particulièrement dynamique qui est devenu une extension du traditionnel downtown san franciscain (situé à l'ouest de Market Street).

Au sud de la Californie, dans le comté d'Orange, l'OCTA et la municipalité d'Anaheim se sont associés pour donner naissance à l'ARTIC qui devrait être inauguré en $2014^{28}$. En 2014, il accueillera les liaisons ferroviaires de Metrolink et d'Amtrak, les bus de l'OCTA et les navettes du Anaheim Resort Transit. Dans les années 2020, cette infrastructure devrait également devenir la gare du train à grande vitesse. Le site choisi se situe à proximité de l'Interstate 5 et est avant tout tourné vers les activités touristiques et de loisirs avec le parc de Disneyland, le centre de convention, le stade des Angels, un centre commercial et des hôtels. Des résidences récentes ont été construites, mais sont encore en grande partie vides. Le réseau de transport est plutôt dense dans cet espace puisque les bus et les navettes assurent des liaisons de la gare vers le parc et le centre de convention. Disneyland a mis en place un réseau de transport privé entre le parc lui-même et ses extensions, notamment les parkings (navettes spéciales pour les visiteurs et pour les employés à partir des différents parkings du parc). Les hôtels assurent également un service de navette pour leurs clients vers le parc. On assiste donc à un véritable ballet de navettes et

\footnotetext{
${ }^{28}$ Voir le site du projet : www.articinfo.com
} 
de bus à l'entrée du parc, interdit aux véhicules privés. Avec l'arrivée de la grande vitesse, défendue par les élus locaux, une réorganisation du réseau de transports locaux serait envisageable, notamment un renforcement des bus et des navettes à partir de la gare ARTIC et la création d'un tramway entre l'ARTIC et le parc Disneyland.

Le dernier terrain d'étude est celui du centre de Los Angeles autour de la gare d'Union Station. Cette gare est scindée en deux pôles : la gare Amtrak au sud et la gare Metrolink/Metro au nord. Le projet de réhabilitation de la gare, actuellement à l'étude, devait être présenté au conseil d'administration de Metro Los Angeles en septembre $2013^{29}$. Le quartier ne devrait quant à lui pas subir de restructuration majeure avec l'arrivée du train à grande vitesse. Des réhabilitations mineures ont eu lieu et sont encore en cours sur une portion très limitée de downtown, entre First Street et Second Street. L'élément architectural le plus connu de cette réhabilitation est le Walt Disney Concert Hall, réalisé par l'architecte Frank Gehry. Dans la même veine, le Broad est actuellement en construction afin d'accueillir un tout nouveau musée d'art contemporain. La réhabilitation, certes timide, du downtown de Los Angeles replace donc les établissements culturels au centre de la ville, alors qu'ils étaient dispersés auparavant (le Getty Center sur les collines de Santa Monica). Toutefois, cette réhabilitation très limitée se concentre sur trois blocs et l'est de downtown est par ailleurs totalement délaissé. Le contraste entre l'ouest de downtown et ses bâtiments de bureaux et ses hôtels et les friches urbaines de l'est reste marqué. Les pôles d'attractivité touristique de Los Angeles ne sont en effet pas dans downtown, même si le Walt Disney Concert Hall et le Pueblo attirent quelques touristes, ceux-ci se ruent plutôt dans les quartiers d'Hollywood, de Beverly Hills et les plages de Santa Monica ou de Venice. Toutefois, l'arrivée du train à grande vitesse pourrait redynamiser les projets de réhabilitation urbaine dans downtown et donner un second souffle à ce quartier à l'image de South of Market à San Francisco.

\section{Conclusion}

Le projet californien de grande vitesse prend pour appui et fondement un discours ambitieux et un terrain favorable à son développement. La Californie, urbaine et touristique, apparaît en effet comme un des États les plus enclins au développement d'une telle infrastructure aux États-Unis. L'analyse de ce discours et sa mise à 1 'épreuve des faits laissent penser que la grande vitesse pourrait s'affirmer comme un accélérateur et un révélateur de nouvelles pratiques, tant

\footnotetext{
${ }^{29}$ Voir le site de Metro Los Angeles : http://www.metro.net/projects/
} LA-union-station/ en termes de mobilités que de territoires. Enfin, ce sont aux échelles urbaines et infra-urbaines que les attentes sont les plus importantes. La future desserte à grande vitesse pourrait engendrer un renforcement des réseaux urbains californiens, mais également un désenclavement de certains territoires urbains du centre de la Californie. La grande vitesse permettrait par ailleurs une revalorisation des centres urbains californiens par l'implantation de gares nouvelles ou remodelées dans des tissus urbains en mutation. Les centres urbains (downtown) et les gares n'étant pas des éléments classiques et symboliques des paysages urbains californiens, la grande vitesse pourrait provoquer la formation d'une nouvelle identité urbaine.

Ces impacts urbains et architecturaux placent le projet californien sous le signe de l'originalité. Si de nombreuses villes nord-américaines tentent de réhabiliter leurs centres depuis plusieurs années par des opérations de revitalisation des fronts de mer (Baltimore) ou de restructuration de quartiers industriels (SOMA à San Francisco), les infrastructures ferroviaires n'étaient plus considérées comme un levier de développement urbain et de modernité architecturale aux États-Unis depuis l'entre-deux-guerres (Union Station à Los Angeles). Au-delà des impacts urbains, qui sont d'ores et déjà présents dans les projets et les débats municipaux, la Californie se distingue des autres États nord-américains par la précocité, l'avance et l'originalité de son projet. Alors que les critiques se multiplient et que le soutien fédéral s'affaiblit en raison de l'impopularité de l'administration Obama, la Californie maintient malgré tout son projet. Elle envisage même dès aujourd'hui l'extension de sa ligne vers Las Vegas ou Salt Lake City, imaginant la ligne à grande vitesse californienne comme le segment initial de la grande vitesse dans l'Ouest américain.

\section{Bibliographie}

1. Bazin S, Beckerich C, Delaplace M (2010) Desserte à grande vitesse, activation des ressources spécifiques et développement du tourisme : le cas de l'agglomération rémoise. Belgeo 1-2: 65-77.

2. Urena Frances JM, Ribalaygua Batalla C (2004) Le réseau ferroviaire à grande vitesse espagnol : état actuel d'avancement et d'appropriation par les villes desservies. Transports Urbains 106: 3-10.

3. California High-Speed Rail Authority (2012) California HighSpeed Rail Program Revised 2012 Business Plan, Building California's Future. Sacramento, California High-Speed Rail Authority, $212 \mathrm{p}$.

4. Perl A (2002) New Departures, Rethinking Rail Passenger Policy in the Twenty-First Century. Lexington, The University Press of Kentucky, $334 \mathrm{p}$.

5. Foucrier A (2010) Le mythe californien dans l'histoire américaine. Pouvoirs 133: 5-15.

6. Starr K (1985) Inventing the Dream, California through the Progressive Era. Oxford University Press, Oxford/New York, $380 \mathrm{p}$. 
7. Thompson LS (1994) High-Speed Rail (HSR) in the United StatesWhy Isn't There More? Japan Railway \& Transport Review 3: 32-39.

8. Clifford Black R (2005) The Acela Express. Japan Railway \& Transport Review 40:18-21.

9. Barron I (2009) High Speed lines in the World. UIC High Speed Department, $28 \mathrm{p}$.

10. Catz SL, Christian A (2010) Thinking Ahead, High-Speed Rail in Southern California. UC Irvine, Institute of Transportation Studies, Irvine, $28 \mathrm{p}$.

11. Lane BW (2012) On the utility of high-speed rail in the United States. Journal of Transport Geography 22: 282-284.

12. United States Department of Transportation (2010) High-Speed Intercity Rail Program-California, Washington DC, US Department of Transportation, $11 \mathrm{p}$.

13. Federal Railroad Administration (2013) High-Speed Intercity Passenger Rail Program, Federal Investment Highlights. United States Department of Transportation, Washington DC, 2 p.

14. The NEC Master Plan Working Group (2010) The Northeast Corridor Infrastructure Master Plan, 178 p.

15. Federal Railroad Administration (2013) High-Speed Intercity Passenger Rail Program, Northeast \& Southeast Region Investments (2009-11). United States Department of Transportation, Washington DC, $1 \mathrm{p}$.

16. Midwest High Speed Rail Association (2011) The Economic Impacts of High Speed Rail: Transforming the Midwest. Chicago, $6 \mathrm{p}$.

17. Xpress West (2012) Xpress West Media Kit. 18 p.

18. Western High Speed Rail Alliance (2012) Fact Sheet \& Brochure. $7 \mathrm{p}$.

19. Federal Railroad Administration (2011) High-Speed Intercity Passenger Rail Program, California \& Northwest Region Investments (2009-11). United States Department of Transportation, Washington DC, $1 \mathrm{p}$.

20. Johnson BE (2012) American intercity passenger rail must be truly high-speed and transit-oriented. Journal of Transport Geography 22: 295-296.

21. California High-Speed Rail Authority (2011) California HighSpeed Rail Program Draft 2012 Business Plan. Sacramento, California High-Speed Rail Authority, $230 \mathrm{p}$.

22. Flyvbjerg B (2009) Survival of the unfittest: why the worst infrastructure gets built-and what we can do about it. Oxford Review of Economic Policy 25-3: 344-367.

23. Ryder A (2012) High Speed Rail. Journal of Transport Geography 22: 303-305.

24. California High-Speed Rail Authority (2012) California HighSpeed Train, Revised Draft EIR/Supplemental Draft EIS, Fresno to Bakersfield. Sacramento, California High-Speed Rail Authority, $2133 \mathrm{p}$.

25. California High-Speed Rail Authority (2012) California HighSpeed Train, Final Merced to Fresno Section, Project EIR/EIS. Sacramento, California High-Speed Rail Authority, 1645 p.

26. Button K (2012) Is there any economic justification for high-speed railways in the United States? Journal of Transport Geography 22: 300-302.

27. Orange County Business Council (2008) The Economic Impact of High Speed Trains for Orange County. $26 \mathrm{p}$.

28. Levinson DM (2012) Accessibility impacts of high-speed rail. Journal of Transport Geography 22: 288-291.
29. California High-Speed Rail Authority (2010) California HighSpeed Train, Project Environmental Impact Report/Environmental Impact Statement, Preliminary Palmdale to Los Angeles Section, Alternative Analysis Report, Volume 1. Sacramento, California High-Speed Rail Authority, $151 \mathrm{p}$.

30. California High-Speed Rail Authority (2011) California HighSpeed Train, Preliminary Alternatives Analysis Report, Los Angeles to San Diego via the Inland Empire Section. Sacramento, California High-Speed Rail Authority, 122 p.

31. Bazin S, Beckerich C, Blanquart C, Delaplace M, Vandenbossche L (2011) Grande vitesse ferroviaire et développement économique local : une revue de la littérature. Colloque de l'ASRDLF : Identité, Qualité et Compétitivité Territoriale - Développement économique et cohésion dans les territoires alpins, Aoste : Italie (2010), 23 p.

32. Gourvish T (2010) The High-Speed Rail Revolution: History and Prospects. HS2, Department of Transport, Londres, $44 \mathrm{p}$.

33. Gravari-Barbas M, Ripoll F (2010) De l'appropriation à la valorisation, et retour. Norois 217: 7-12.

34. Ripoll F, Veschambre V (2005) L'appropriation de l'espace comme problématique. Norois 195: 7-15.

35. Douzet F (2007) La Couleur du pouvoir, Géopolitique de l'immigration et de la ségrégation à Oakland, Californie. Belin, collection « Histoire \& Société », Paris, 383 p.

36. Ghorra-Gobin C (1997) Los Angeles, Le mythe américain inachevé. CNRS Éditions, Paris, $311 \mathrm{p}$.

37. California High-Speed Rail Authority (2012) Bay Area to Central valley High-Speed Train, Partially Revised Final Program, Environmental Impact Report. Sacramento, California High-Speed Rail Authority, 492 p.

\section{Annexe à la bibliographie}

\section{Liste des acteurs rencontrés en juin 2012 et cités dans l'article}

Sylvia Ballin, Maribel de la Torre et Mario Hernandez : membres du conseil municipal de San Fernando.

TimBen Boydston, Frank Ferry, Marsha MacLean et Laurene Weste : membres du conseil municipal de Santa Clarita.

Lucy Dunn : présidente du Orange County Business Council.

Stephen G. Fox et son équipe : responsable de l'aménagement des transports pour le SCAG.

Bob Kellar : maire de Santa Clarita.

Jamie Lai et son équipe : responsable de l'aménagement des transports pour la municipalité d'Anaheim.

Antonio Lopez : maire de San Fernando.

Don Sepulveda : responsable du transport ferroviaire pour le Los Angeles Metro.

Ralph Vartabedian : journaliste au Los Angeles Times. 